\title{
Terminology/Terminologie
}

\section{Nomenclature for secreted regulatory proteins of the immune system (interleukins)*}

\author{
WHO-IUIS Nomenclature Subcommittee on Interleukin Designation'
}

The recommended procedures and criteria for interleukin designations are described. The offically adopted designations are, in sequence, from interleukin-1 to interleukin-10, including interleukin-1 $\alpha$ and interleukin-1 $\beta$.

\section{Criteria for designations}

The following procedures are recommended by a Subcommittee of the Nomenclature Committee of the International Union of Immunological Societies (IUIS) for the naming of new secreted regulatory proteins of the immune system (i.e., lymphokines, cytokines, interleukins). If the discoverers of such a molecule wish to utilize an interleukin designation (i.e., interleukin-n), they are urged not to do so unilaterally but to apply to the newly established Standing Committee on Interleukin Designation of IUIS for approval. ${ }^{a}$ The granting of such an interleukin designation would depend on whether the following criteria have been met:

(1) The molecule shall have been purified, molecularly cloned and expressed. Its nucleotide and inferred amino acid sequence should be distinct

\footnotetext{
* This article was drafted by a group of experts working under the auspices of the International Union of Immunological Societies (IUIS) and has been approved by the Nomenclature Committee of IUIS. A French translation appears on pages 485-486. Requests for reprints and all correspondence should be addressed to the Chairman of the IUIS Nomenclature Committee, Dr Michel Kazatchkine, Unité d'Immunopathologie, Hôpital Broussais, 96 rue Didot, 75014 Paris, France.

1 Members of the Nomenclature Subcommittee: W.E. Paul (USA) (Chairman), Tadamitsu Kishimoto (Japan), F. Melchers (Switzerland), D. Metcalf (Australia), T. Mosmann (Canada), J. Oppenheim (USA), Nancy Ruddle (USA), and J. Van Snick (Belgium).

a Standing Committee on Interleukin Designation: Chairman: T. Mosmann, Department of Immunology, University of Alberta, Canada.
}

Reprint No. 5199 from any currently known interleukin or indeed from any other already described molecule. The availability of monoclonal antibodies that may neutralize its function would be desirable but not absolutely required, as would information regarding the chromosomal location of the gene encoding the molecule.

(2) The molecule shall have been shown to be a natural product of cells of the immune system, to mediate a potentially important function in immune responses, and preferably also to have more than a single function so that a simple functional name might not be adequate. It is preferred if it could be shown that the predominant actions of the molecule were to mediate functions in the immune system, but it is recognized that this may be difficult to establish. The terms "immune system" and "immune responses" shall be defined very broadly to include leukocytes other than lymphocytes and monocytes as important participants.

(3) If the molecule is a member of an already characterized family of molecules that have their major functions outside the immune system, it would be preferable in most circumstances to use a designation consistent with the naming of other members of the family rather than adopting an interleukin designation.

(4) The Subcommittee in no way wishes to discourage the use of descriptive names for new lymphokines and cytokines. Indeed, it generally recommends that interleukin designations only be sought when the discoverers or other major participants in the field have a compelling reason for seeking such a name. Well-chosen descriptive or 
functional names may have mnemonic value that an interleukin designation is likely to lack.

Based on the proposed requirements for the award of an interleukin designation, it is very unlikely that such a designation could be granted prior to the initial publication describing a new factor. Thus, even if the authors wish that an interleukin designation be awarded, they are urged to choose some provisional name. If they wish, they may indicate in their publications that they intend to apply for an interleukin designation, without specifying what that designation may be.

In a situation in which more than a single research group was involved in the discovery of the molecule or in some very important aspects of the characterization of the molecule, it is desirable that agreement be obtained from all the major contributors prior to the application for an interleukin designation. If an interleukin designation is sought for a molecule that has been studied by several groups for an extended period of time, it is likely that the Standing Committee will recommend that some type of consensus on the part of all concerned be obtained prior to application for an interleukin designation.

The Standing Committee may wish to obtain expert advice regarding the suitability of awarding an interleukin designation to a particular molecule. Accordingly, supporting material regarding the characterization of the potential interleukin must be in the public domain and, preferably, should have been published in refereed journals.

\section{Designations}

A list of official interleukin designations will be sent to journals that regularly publish papers in this field. The editors will be asked to request their authors not to use non-official interleukin designations. Indeed, once this mechanism is in place, the immunological community is strongly urged not to unilaterally adopt interleukin designations in order to avoid the confusion that may occur when several molecules are given the same designation or when molecules whose principal functions lie outside the immune system receive an interleukin designation.

Currently accepted interleukin designations officially adopted by the IUIS Nomenclature Committee are listed below:

Interleukin-l $\alpha$; Interleukin-l $\beta$

Interleukin-2

Interleukin-3

Interleukin-4

Interleukin-5

Interleukin-6

Interleukin-7

Interleukin-8

Interleukin-9

Interleukin-10. 


\title{
Nomenclature des protéines régulatrices sécrétoires du système immunitaire (interleukines)*
}

\author{
Sous-Comité de nomenclature OMS-UISI pour les interleukines'
}

On trouvera décrits ci-dessous le mode et les critères de désignation recommandés pour les interleukines. La terminologie officiellement adoptée couvre les interleukines 1 à 10, y compris les interleukines $1 \alpha$ et $1 \beta$.

\section{Critères}

Le Sous-Comité de nomenclature de l'Union internationale des Sociétés d'Immunologie (UISI) recommande d'appliquer la procédure suivante à la désignation des protéines régulatrices secrétoires du système immunitaire nouvellement découvertes (lymphokines, cytokines, interleukines). Si l'on souhaite donner à une molécule nouvellement découverte l'appellation d'interleukine (interleukine $\mathrm{n}$ par exemple) il est fortement recommandé de ne pas prendre cette décision unilatéralement, mais de solliciter l'accord du "Comité permanent de l'UISI pour la nomenclature des interleukines" qui vient de se créer. ${ }^{a}$ L'attribution du terme d'interleukine est soumise aux critères suivants:

1) La molécule doit avoir été purifiée, son gène cloné et exprimé. Sa séquence nucléotidique et la séquence primaire des acides aminés codés doivent être distinctes de celles des interleukines connues, et même de toutes les molécules déjà décrites. Il est souhaitable, sans obligation cependant, que l'on connaisse des anticorps monoclonaux capables de neutraliser l'activité fonctionnelle de la molécule; il en est de même pour l'information sur la localisation chromosomique du gène codant pour la molécule.

\footnotetext{
* Cet article a été rédigé par un groupe d'experts réunis sous le patronage de l'Union internationale des Sociétés d'Immunologie (UISI) et a été approuvé par son Comité de nomenclature. L'original anglais figure dans ce même Bulletin pages 483-484. Tirés à part et correspondance: Dr Michel Kazatchkine, Président du Comité de nomenclature de I'UISI, Unité d'Immunopathologie, Hôpital Broussais, 96 rue Didot, 75014 Paris (France).

1 Membres du Sous-Comité de nomenclature: W.E. Paul (EtatsUnis d'Amérique) (Président); Tadamitsu Kishimoto (Japon); F. Melchers (Suisse); D. Metcalf (Australie); T. Mosmann (Canada); J. Oppenheim, Nancy Ruddle (Etats-Unis d'Amérique); J. Van Snick (Belgique).

a Comité permanent pour la nomenclature des interleukines: T. Mosmann, Président, Départment d'Immunologie, Université de l'Alberta (Canada).
}

2) La molécule doit avoir les propriétés suivantes: être naturellement sécrétée par les cellules du système immunitaire, intervenir comme médiateur dans un processus potentiellement important de la réponse immunitaire et avoir de préférence plusieurs fonctions, de sorte qu'une terminologie faisant référence à une fonction unique risquerait d'être inappropriée. Il est préférable que les fonctions principales de la molécule soient celles d'un immunomédiateur, ce que le Comité estime cependant difficile à établir. La définition des expressions "système immunitaire" et "réponse immunitaire" doit être assez large pour inclure d'autres leucocytes que les lymphocytes et les monocytes parmi les éléments importants des processus immunitaires.

3) Si la molécule s'avère appartenir à une famille déjà caractérisée de molécules dont les fonctions principales ne concernent pas le système immunitaire, il est le plus souvent souhaitable de donner une appellation correspondant aux appellations des autres membres de la famille, plutôt que d'adopter le nom d'interleukine.

4) Le Sous-Comité ne souhaite en rien décourager l'utilisation de noms descriptifs pour désigner les nouvelles lymphokines et cytokines. Il recommande, en effet, de ne recourir au terme d'interleukine que quand le découvreur ou d'autres experts dans ce domaine ont des raisons impératives de lui donner une telle appellation. Un nom bien choisi, de type descriptif ou fonctionnel, peut avoir un intérêt mnémotechnique qui risque de manquer à "interleukine".

Considérant les critères proposés pour l'attribution du nom d'interleukine à une substance, il est très peu probable qu'une nouvelle molécule puisse recevoir cette appellation avant la première publication de sa description. Aussi est-il vivement recommandé aux auteurs souhaitant que la nouvelle molécule soit appelée interleukine de lui donner un nom provisoire. Ils pourront indiquer leur souhait dans leur publication, sans toutefois envisager d'appellation précise. 
Dans le cas où plusieurs groupes de recherche sont impliqués dans la découverte de la molécule ou de certaines de ses caractéristiques particulièrement importantes, l'accord préalable des collaborateurs principaux est souhaitable avant la demande d'appellation "interleukine". Si l'appellation est destinée à une molécule qui a fait l'objet de longues études par plusieurs groupes de recherche, le Comité permanent recommandera très probablement que les experts s'accordent sur les caractéristiques de cette molécule avant de faire leur demande.

Le Comité permanent souhaitera peut-être obtenir l'avis d'experts sur l'opportunité de la désignation d'une molécule par le terme d'interleukine. En conséquence, les données concernant la caractérisation de cette molécule doivent être du domaine public, et de préférence, avoir été publiées dans des revues ayant un comité de lecture.

\section{Terminologie}

La liste des appellations officielles applicables aux interleukines sera adressée aux revues qui publient régulièrement des articles dans ce domaine. La rédaction sera priée de donner aux auteurs l'instruction d'éviter une nomenclature différente de la nomenclature officielle. En fait, une fois le mécanisme en place, les immunologistes sont invités à ne pas employer la terminologie des interleukines sans consulter le Comité, afin d'éviter la confusion qui pourrait résulter de l'utilisation du même nom pour plusieurs molécules ou de l'attribution du terme d'interleukine à des molécules dont les fonctions principales s'exercent hors du système immunitaire.

La liste qui suit est celle des interleukines dont la désignation a été officiellement adoptée par le Comité de nomenclature de l'UISI:

Interleukine $1 \alpha$; interleukine $1 \beta$

Interleukine 2

Interleukine 3

Interleukine 4

Interleukine 5

Interleukine 6

Interleukine 7

Interleukine 8

Interleukine 9

Interleukine 10 . 